\title{
Corrigendum \\ Neuroimaging-Aided Prediction of the Effect of \\ Methylphenidate in Children with Attention-Deficit Hyperactivity Disorder: A Randomized Controlled Trial
}

\begin{abstract}
Ayaka Ishii-Takahashi, Ryu Takizawa, Yukika Nishimura, Yuki Kawakubo, Kasumi Hamada, Shiho Okuhata, Shingo Kawasaki, Hitoshi Kuwabara, Takafumi Shimada, Ayako Todokoro, Takashi Igarashi, Kei-ichiro Watanabe, Hidenori Yamasue, Nobumasa Kato, Kiyoto Kasai and Yukiko Kano
\end{abstract}

Neuropsychopharmacology (20I5) 40, 2852; doi:I 0.I038/npp.20I5.I54; published online 8 July 2015

Correction to: Neuropsychopharmacology (2015) advance online publication 27 May 2015; doi:10.1038/npp.2015.128
A Supplementary Information file containing extraneous information was unintentionally published for this article and was removed post-publication. We regret the error. 